\title{
ADUBAÇÃO FOSFATADA EM CULTIVARES DE ALFACE CULTIVADA EM SOLOS DEFICIENTES
}

\author{
COUTINHO, Edson Luiz Mendes ${ }^{94}$ \\ CARVALHO, Flávio Paranhos de ${ }^{95}$ \\ FRANCO, Henrique Coutinho Junqueira ${ }^{2}$ \\ ORIOLI JÚNIOR, Valdeci ${ }^{96}$ \\ COUTINHO NETO, André Mendes ${ }^{97}$ \\ UETA, Fabiano Zancaner ${ }^{2}$
}

Recebido em: 2008-06-09

Aprovado em: 2008-08-28

ISSUE DOI: $10.3738 / 1982.2278 .87$

\begin{abstract}
RESUMO: Não obstante a carência de pesquisa com a cultura da alface no estado de São Paulo, são recomendadas doses extremamente elevadas de fósforo $(\mathrm{P})$, sem levar em consideração o tipo ou cultivar. Sendo assim, conduziu-se em casa de vegetação, um experimento com o objetivo de verificar os efeitos da adubação fosfatada nas concentrações de $\mathrm{P}$ no solo e na planta, assim como, na produção de massa seca da parte aérea da alface. Empregou-se o delineamento experimental inteiramente casualizado, com três repetições, segundo um arranjo fatorial $5 \times 2 \times 3$ (cinco doses de $\mathrm{P}$, dois solos e três cultivares). As doses $0,25,50,100$ e $150 \mathrm{mg} \mathrm{kg}^{-1}$ de $\mathrm{P}$ foram aplicadas na forma de fosfato monoamônio (MAP). Os cultivares Regina (tipo lisa), Verônica (tipo crespa) e Lucy Brown (tipo americana) foram cultivados, por 48 dias, em amostras dos solos: Latossolo Vermelho-Amarelo (LVA) e Argissolo Vermelho-Amarelo (PVA). Verificou-se que a adubação fosfatada aumentou significativamente a produção de massa seca das plantas, sendo o cultivar Lucy Brown o mais produtivo. As plantas de alface cultivadas no LVA apresentaram maior produção de massa seca que as cultivadas no PVA. As doses de $\mathrm{P}$ aumentaram as concentrações do nutriente no solo e na planta. Entre os cultivares, entretanto, não houve diferença significativa nas concentrações de P na parte aérea. As plantas nos tratamentos que não receberam fósforo apresentaram sintomas de deficiência (teores de $\mathrm{P}$ na parte aérea inferiores a $2,3 \mathrm{~g} \mathrm{~kg}$ ${ }^{1}$ ) e não conseguiram chegar ao final do ciclo.
\end{abstract}

Palavras-chave: Deficiência de P. Fósforo disponível. Lactuca sativa L. Solo tropical. Tipos de alface.

\section{RESPONSE OF LETTUCE CULTIVARS TO PHOSPHORUS FERTILIZATION IN DEFICIENT SOILS}

\footnotetext{
${ }^{94}$ Professor Titular. UNESP - Campus de Jaboticabal. E-mail: coutinho@ fcav.unesp.br 95Engo . Agr ${ }^{\circ}$., Ex-estagiário do Departamento de Solos e Adubos. UNESP - Campus de Jaboticabal ${ }^{96}$ Mestrando em Agronomia (Produção Vegetal). UNESP - Campus de Jaboticabal.

${ }^{97}$ Acadêmico do Curso de Agronomia da FE/FAFRAM
} 
SUMMARY: A greenhouse experiment was carried out with the objective to evaluate the response of three lettuce types (cultivars: Regina, Verônica, and Lucy Brown) grown on two soils (Paleudult and Hapludox), to phosphorus fertilization. It was used a complete randomized design with three replications of treatments in a factorial arrangement $5 \times 3 \times 2$ (five rates of P, three cultivars, and two soils). The rates of phosphorus were: 0 , $25,50,100$, and $150 \mathrm{mg} \mathrm{kg}^{-1}$. Dry matter and phosphorus contents were determined in soil and on plant shoots 48 days after emergency plants. A significant yield response to phosphorus fertilization was observed. The Lucy Brown cultivar was more productive than 'Regina' and 'Verônica'. The letucce grown on Hapludox tended to have higher yield of dry matter. Among the cultivars, it was not verified significant differences in the $\mathrm{P}$ concentrations in plant shoots. The appearing of phosphorus deficiency symptoms in lettuce were associated with $\mathrm{P}$ contents on plant shoots lower than $2.3 \mathrm{~g} \mathrm{~kg}^{-1}$.

Keywords: Available phosphorus. Lactuca sativa L. Lettuce types. P deficiency. Tropical soil.

\section{INTRODUÇÃO}

A alface (Lactuca sativa L.) é uma das hortaliças mais difundidas no mundo, possuindo grande importância na alimentação humana (pois é fonte de minerais e celulose) e constituindo-se a mais popular dentre aquelas em que as folhas são consumidas.

A alface é considerada uma planta não facilmente adaptável aos solos com baixa disponibilidade de nutrientes na camada arável. Na maioria dos experimentos, essa cultura mostrou uma pronunciada resposta à adição de fósforo, sendo em solos deficientes nesse nutriente requeridas doses superiores a $600 \mathrm{~kg} \mathrm{ha}^{-1}$ de $\mathrm{P}_{2} \mathrm{O}_{5}$ para a máxima produção (SANCHEZ et al. 1990; NAGATA et al. 1992; SANCHES; EL-HOUT, 1995; MOTA et al., 2003).

O grau de resposta à adubação fosfatada, entretanto, é dependente do teor de $\mathrm{P}$ no solo. Assim, em Histosolos da Flórida, a recomendação desse nutriente é realizada quando o teor de P solúvel em água está abaixo do nível crítico de $22 \mathrm{mg} \mathrm{dm}^{-3}$ (SANCHEZ; BURDINE, 1988). Costigan; Heaviside (1988) não verificou aumento na produção de alface em solos com concentrações de P superiores a $100 \mathrm{mg} \mathrm{dm}^{-3}$ (extrator Olsen). No estado de São Paulo, Seno; Koga (1993), empregando doses crescentes de superfosfato simples e húmus de minhoca, também não observaram incrementos significativos na produção, cultivando alface em um solo com teor de $\mathrm{P}$ (resina) de $55 \mathrm{mg} \mathrm{dm}^{-3}$.

Não obstante a carência de pesquisa para essa cultura no estado de São Paulo, são recomendadas doses extremamente elevadas de fósforo, sem levar em consideração o tipo ou 
cultivar (TRANI et al. 1996). Deve-se mencionar, entretanto, que existem evidências de variações entre e dentre espécies quanto ao uso e acúmulo de fósforo (BUSO; BLISS, 1988; OTANI; AE, 1996) e do controle genético dessas características. Dessa maneira, pode ocorrer variação entre os cultivares na eficiência da aquisição de $\mathrm{P}$ do meio ambiente e o uso eficiente desse nutriente, ou seja, a capacidade de converter P em biomassa ou produção. Embora informações limitadas tenham demonstrado exigências nutricionais diferenciadas entre os cultivares (GARCIA et al. 1982), não existe informação disponível na literatura nacional sobre o comportamento dos tipos de alface à adubação fosfatada. Sendo assim, o presente trabalho teve por objetivo verificar os efeitos da adubação fosfatada no estado nutricional, no solo e na produção de matéria seca de três tipos de alface, cultivadas em dois solos deficientes em P.

\section{MATERIAL E MÉTODOS}

O experimento foi realizado em casa de vegetação, em vasos de cerâmica preenchidos com 3,8 kg de amostras de dois solos, coletadas da camada arável $(0-20 \mathrm{~cm})$ : Argissolo Vermelho-Amarelo distrófico (PVAd), textura arenosa/média, proveniente do município de Pindorama - SP; e um Latossolo Vermelho-Amarelo distrófico (LVAd), textura média, coletado no município de São Carlos - SP.

Os principais atributos químicos para fins de fertilidade do solo são apresentados na Tabela 1.

Tabela 1: Caracterização química dos solos estudados* .

\begin{tabular}{|c|c|c|c|c|c|c|c|c|c|}
\hline Solo & $\mathrm{pH}$ & M.O. & $\mathrm{P}$ (Resina) & $\mathrm{K}$ & $\mathrm{Ca}$ & $\mathrm{Mg}$ & $\mathrm{H}+\mathrm{Al}$ & CTC & $\mathrm{V}$ \\
\hline & & $\mathrm{g} \mathrm{dm}^{-3}$ & $\mathrm{mg} \mathrm{dm}^{-3}$ & ---- & ---- & $\mathrm{amol}_{\mathrm{c}} \mathrm{c}$ & & & $\%$ \\
\hline PVAd & 5,6 & 31,0 & 3 & 3,5 & 48 & 12 & 22,6 & 86,1 & 74 \\
\hline LVAd & 5,5 & 26,0 & 11 & 2,0 & 39 & 13 & 19,9 & 73,9 & 73 \\
\hline
\end{tabular}

* análises realizadas segundo métodos descritos por RAIJ et al. (1987).

Foi utilizado o delineamento experimental inteiramente casualizado, com três repetições, segundo um arranjo fatorial $5 \times 2 \times 3$ (cinco doses de fósforo, dois solos e três cultivares), resultando em um total de 90 vasos.

As doses de fósforo utilizadas na forma de fosfato monoamônio p.a. (MAP) foram: 0, 25, 50, 100 e $150 \mathrm{mg} \mathrm{kg}^{-1}$ de P. Os cultivares foram Regina (tipo lisa), Verônica (tipo crespa) 
e Lucy Brown (tipo americana). Todos os tratamentos receberam no plantio uma adubação constante com: $150 \mathrm{mg} \mathrm{kg}^{-1}$ de $\mathrm{K}$ (cloreto de potássio p.a.), $3 \mathrm{mg} \mathrm{kg}^{-1}$ de $\mathrm{Zn}$ (sulfato de zinco p.a.) e $1,5 \mathrm{mg} \mathrm{kg}^{-1} \mathrm{de} \mathrm{B}$ (ácido bórico p.a.). Deve-se mencionar ainda, que o fosfato monoamônio atuou também como fonte de $\mathrm{N}$, assim, o que faltou para integralizar a dose deste macronutriente na semeadura $\left(70 \mathrm{mg} \mathrm{kg}^{-1} \mathrm{de} \mathrm{N}\right)$ foi complementado com nitrato de amônio p.a. Todos estes fertilizantes, na forma de solução, foram misturados ao volume total do solo. Posteriormente, adicionou-se água destilada, deixando-se o solo de cada vaso incubado com os sais adicionados, por um período de 20 dias, mantendo-se os solos com $80 \%$ de sua capacidade máxima de retenção de água. Após esse período, os solos foram deixados para secar, coletando-se amostra de solo de cada tratamento (300g de solo seco ao ar).

Por ocasião da semeadura, foram empregadas dez sementes de alface por vaso, realizando-se o desbaste sete dias após a emergência, deixando-se uma planta por vaso.

Foram efetuadas duas coberturas nitrogenadas com $75 \mathrm{mg} \mathrm{kg}^{-1}$ de $\mathrm{N}$ por cobertura, aos 15 e 30 dias após a emergência das plantas. Utilizou-se como fonte de $\mathrm{N}$ o sulfato de amônio e o nitrato de amônio, na primeira e segunda cobertura, respectivamente.

Durante o período de condução do experimento foram realizadas regas com água destilada, procurando-se através de pesagens diárias, manter os solos com $80 \%$ da sua capacidade máxima de retenção de água.

Após a colheita, que foi realizada 48 dias após a emergência das plantas, a parte aérea foi lavada com água destilada, seca em estufa a $65^{\circ} \mathrm{C}$ e, após a secagem, foi pesada para determinação da massa seca. Posteriormente, o material foi moído e armazenado. Cumpre salientar que, as plantas do tratamento com a ausência de fósforo não conseguiram chegar ao final do ciclo, sendo assim colhidas antes.

Para determinação das concentrações de P no tecido vegetal e no solo, as amostras foram preparadas e analisadas segundo Bataglia et al. (1983) e Raij et al. (1987), respectivamente.

A análise estatística foi realizada segundo Banzatto; Kronka (1995).

\section{RESULTADOS E DISCUSSÃO}

A adubação fosfatada proporcionou incrementos significativos nas concentrações de $\mathrm{P}$ no solo $\left(\mathrm{F}=2.073,40^{* *}\right)$ e na parte aérea das plantas de alface $\left(\mathrm{F}=356,18^{* *}\right)$. 
Nas plantas dos tratamentos que não receberam a aplicação de $\mathrm{P}$, foram observados, já nos estádiosiniciais de desenvolvimento da cultura, sintomas típicos de carência desse nutriente. Os sintomas observados, em todos os cultivares, eram semelhantes aos descritos por Katayama (1993), ou seja, as plantas apresentavam um baixo desenvolvimento vegetativo, com as folhas mais velhas exibindo inicialmente uma coloração verde-opaca, evoluindo, posteriormente, para uma coloração vermelho-bronze. Essas plantas não conseguiram completar o ciclo, sendo colhidas precocemente. As concentrações de P nestas plantas estiveram abaixo dos níveis críticos determinados por Geraldson et al. (1973), Beverely (1984), Sanchez et al. (1988) e Sanchez et al. (1990).

Verificou-se que os teores de P eram mais elevados no LVA, independentemente da dose de P utilizada. Estes dados podem ser visualizados na Figura 1, a qual foi elaborada com base no desdobramento da interação significativa dose x solo.

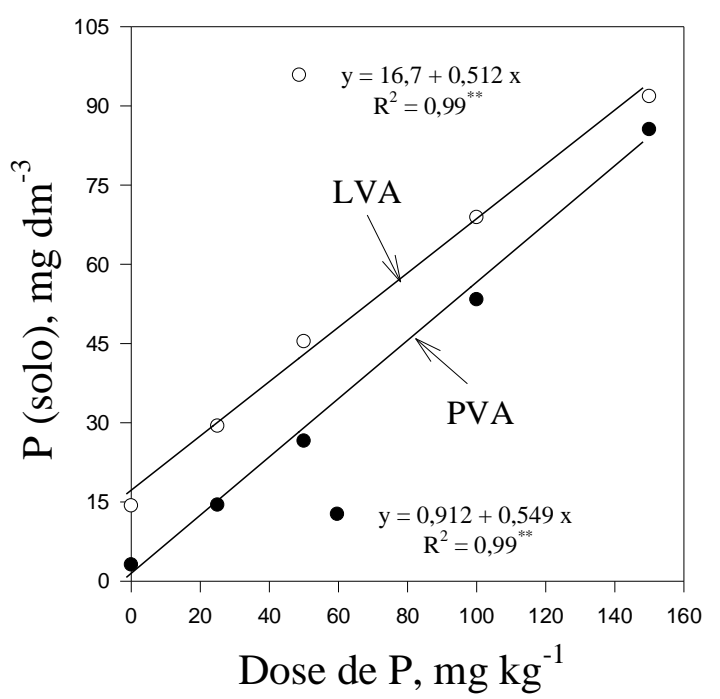

Figura 1: Efeitos da adubação fosfatada nos teores de $\mathrm{P}$ nos solos Agissolo VermelhoAmarelo (PVA) e Latossolo Vermelho-Amarelo (LVA).

Esse resultado pode ser atribuído ao teor inicial de $\mathrm{P}$ mais elevado nesse solo, conforme observado na Tabela 1. É importante salientar que, para ambos os solos, a dose de $150 \mathrm{mg} \mathrm{kg}^{-1}$ de $\mathrm{P}$ proporcionou teores de $\mathrm{P}$ (Figura 1) que podem ser enquadrados dentro da classe de teores altos para as hortaliças (RAIJ et al. 1996). 
Por outro lado, quando se utiliza solo distinto é interessante procurar saber como as doses de $\mathrm{P}$ aplicadas se refletem na análise química do solo. Nos dois solos, a relação entre doses e teores de $\mathrm{P}$ foi linear, logo os valores do coeficiente angular e das equações apresentadas na Figura 1, indicam as proporções do P aplicado, recuperadas com o emprego do extrator resina. Observa-se que a eficiência do extrator variou entre os solos, sendo 55 e $51 \%$ do $\mathrm{P}$ aplicado recuperado nos solos PVA e LVA, respectivamente. A variação na recuperação do P entre os solos pode refletir, segundo Novais et al. (2007), a influência de atributos do solo, relacionados ao fator capacidade do nutriente, como teores de argila e de óxidos de ferro e alumínio. Assim, a maior recuperação do P aplicado no PVA pode ser atribuída à menor adsorção desse nutriente, em função das menores proporções de argila e óxidos (Fe e Al).

$\mathrm{O}$ incremento na quantidade de $\mathrm{P}$ no solo se refletiu nas concentrações desse nutriente na parte aérea dos cultivares de alface (Figura 2). Nota-se também, que as maiores concentrações do nutriente nas plantas foram observadas naquelas cultivadas no LVA. Como já mencionado anteriormente, acredita-se que o maior teor inicial de $\mathrm{P}$ nesse solo tenha contribuído para esse resultado.

Embora alguns trabalhos tenham demonstrado que as concentrações de $\mathrm{P}$ nas plantas de alface possam variar com o cultivar (GARCIA et al. 1982; BUSO; BLISS, 1988; SANCHEZ et al. 1988), isto não foi observado no presente experimento (Figura 2).
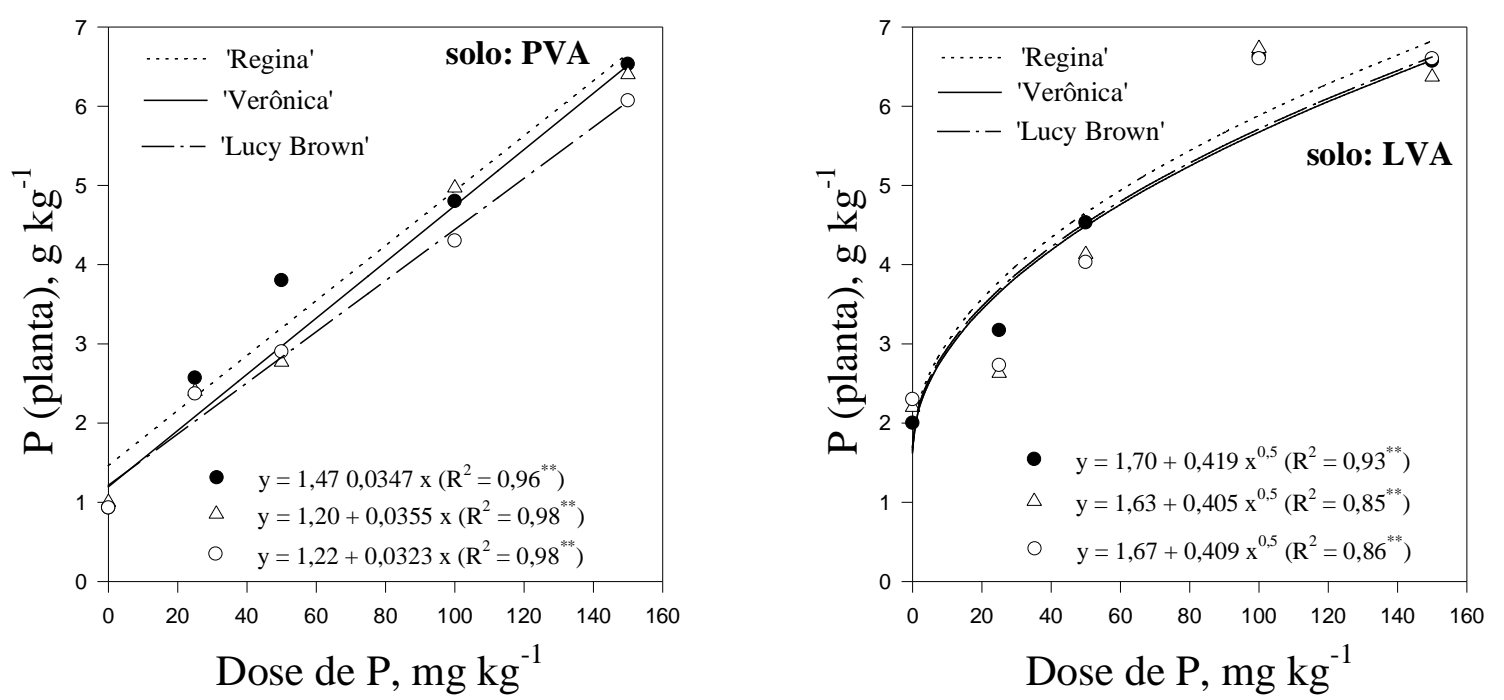

Figura 2: Efeitos da adubação fosfatada nas concentrações de P na parte aérea das plantas de alface cultivada nos solos Agissolo Vermelho-Amarelo (PVA) e Latossolo Vermelho-Amarelo (LVA). 
Considerando-se a faixa de teores adequados de $\mathrm{P}$ nas plantas de alface de 4,0 - 7,0 g $\mathrm{kg}^{-1}$, conforme sugerido por Trani; Raij (1996), verifica-se que no LVA doses de $50 \mathrm{mg} \mathrm{kg}{ }^{-1}$ de $\mathrm{P}$ já proporcionaram concentrações consideradas adequadas desse nutriente na parte aérea dos três cultivares de alface. No PVA, entretanto, foram requeridas doses mais elevadas, a partir de $100 \mathrm{mg} \mathrm{kg}^{-1}$ de P. Relacionando-se a quantidade de P no solo (Figura 1) com os teores de $\mathrm{P}$ na parte aérea dos três cultivares de alface (Figura 2), observa-se que as concentrações desse nas plantas consideradas adequadas ( $\mathrm{P}>4,0 \mathrm{~g} \mathrm{~kg}^{-1}$ ), estiveram associadas a proporções de $\mathrm{P}$ no solo superiores a $45 \mathrm{mg} \mathrm{dm}^{-3}$.

$\mathrm{O}$ aumento das doses de $\mathrm{P}$ promoveu incrementos altamente significativos na produção de matéria seca de alface (Figura 3), corroborando resultados obtidos por Costigan; Heaviside (1988); Sanchez et al. (1990); Nagata et al. (1992); Sanchez; El-Hout (1995); Mota et al. (2003) e Xu et al. (2004).
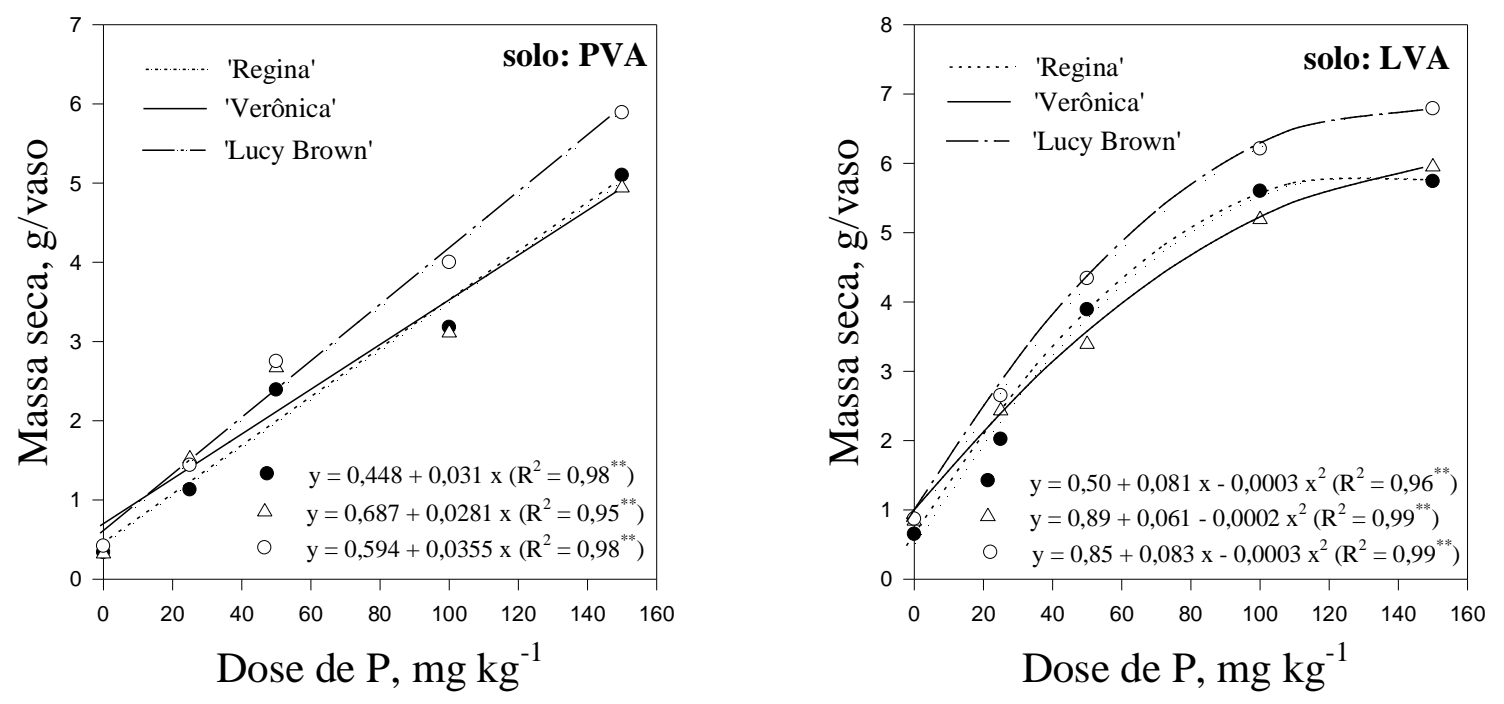

Figura 3: Efeitos da adubação fosfatada na produção dos cultivares de alface nos solos Agissolo Vermelho-Amarelo (PVA) e Latossolo Vermelho-Amarelo (LVA).

Entre os cultivares, a tipo americana (Lucy Brown) foi significativamente $\left(\mathrm{F}=7,90^{* *}\right)$ mais produtiva que as tipos lisa (Regina) e crespa (Verônica). Segundo Mota et al. (2003), isso se deve ao fato da alface tipo americana apresentar um ciclo de desenvolvimento e extração de $\mathrm{P}$ maiores em relação à alface do grupo das folhas soltas lisas ou crespas. 
Em função dos resultados obtidos por Buso; Bliss (1988), onde cultivares de alface podiam apresentar uma eficiência diferente na utilização de $\mathrm{P}$, conforme as concentrações desse nutriente no meio, poder-se-ia esperar um comportamento diferente dos cultivares em função das porções de $\mathrm{P}$ empregadas. Entretanto isto não foi observado, uma vez que a interação cultivares $\mathrm{x}$ doses de $\mathrm{P}$ foi não significativa $(\mathrm{F}=0,90 \mathrm{~ns})$, indicando que não existe a necessidade de ajuste na quantidade de $\mathrm{P}$ em conformidade com o cultivar empregado. Nagata et al. (1992) também verificaram diferenças significativas na produção de matéria seca de cultivares de alface, mas concluíram que a recomendação de adubação para essa cultura não deveria ser modificada, uma vez que os níveis críticos de P no solo, para cada cultivar, eram bastante próximos.

Por outro lado, o efeito das doses de P na produção de matéria seca dentro de cada solo foi diferente (interação dose x solo significativa). Pode ser observado, na Figura 3, que em função do teor inicial de P no LVA ser mais elevado que no PVA, para quantidade de $\mathrm{P}$ inferior a $150 \mathrm{mg} \mathrm{kg}^{-1}$, a produção foi mais elevada quando a alface era cultivada no LVA.

Os resultados em termos de produção de massa seca da parte aérea demonstraram a grande exigência das plantas de alface em $\mathrm{P}$, onde mesmo na presença de altos níveis de $\mathrm{P}$ no solo, incrementos na produção ainda eram observados. Johnstone et al. (2005) conduzindo 12 experimentos em solos com altos teores de $\mathrm{P}$, também verificaram aumentos significativos na produção de alface.

\section{CONCLUSÃO}

A adubação fosfatada aumentou significativamente a produção de matéria seca da parte aérea das plantas de alface, sendo o cultivar Lucy Brown o mais produtivo. Não houve resposta diferenciada dos cultivares às porções de $\mathrm{P}$.

O aparecimento de sintomas de deficiência de fósforo, nas plantas de alface, esteve

associado ás concentrações de $\mathrm{P}$ no solo e na parte aérea inferior a $16 \mathrm{mg} \mathrm{dm}^{-3}$ e $2,3 \mathrm{~g} \mathrm{~kg}^{-1}$, respectivamente.

\section{REFERÊNCIAS}

BANZATTO, D. A; KRONKA, S. N. Experimentação agrícola. Jaboticabal: FUNEP, 1995. $247 \mathrm{p}$. 
BATAGLIA, O. C. et al. Métodos de análise química de plantas. Campinas: Instituto Agronômico, 1983. 48p. (Boletim Técnico, 78)

BEVERELY, R.B. Nutritional survey of the Everglades vegetable industry. In: FLORIDA STATE HORTICULTURAL SOCIETY, v.97, Proceedings... p.201-205, 1984.

BUSO, G. S. C; BLISS, F. A. Variability among lettuce cultivars grown at two levels of available phosphorus. Plant and Soil, v.111, p.67-73, 1988.

COSTIGAN, P. A; HEAVISIDE, M. The effects of starter fertilizer on the early growth and yield of transplanted crisp lettuce on fertile soils. J. Hortic. Sci., v.63, p.247-253, 1988.

GARCIA, L. L. C. et al. Concentração e acúmulo de macronutrientes em alface (Lactuca sativa L.) cv. Brasil 48 e Clause's Aurélia. In: ESCOLA SUPERIOR DE AGRICULTURA LUIZ DE QUEIROZ, Anais... v.34, p.455-485, 1982.

GERALDSON, C. M; KLACAN, G. R; LORENZ, O. A. Plant analysis as an aid for fertilizing vegetable crops. In: WALSH, L.M.; BEATON, J.M. (Eds.) Soil testing and analysis. Madison: Soil Science Society of America, 1973. p.365-379.

JOHNSTONE, P. R. et al. Lettuce response to phosphorus fertilization in high phosphorus soils. HortScience, v.40, p.1576-1586, 2005.

KATAYAMA, N. Nutrição e adubação de alface, chicória e almeirão. In: FERREIRA, M.E; CASTELLANI, P. D; CRUZ, M.C.P. (Eds.) Nutrição e adubação de hortaliças, Piracicaba: Potafos,1993. p.141-148.

MOTA, J. H. et al. Produção de alface americana em função da aplicação de doses e fontes de fósforo. Hort. Brás., v.21, p.620-622, 2003.

NAGATA, R. T; SANCHEZ, C. A; COALE, F. J. Crisphead lettuce cultivar response to fertilizer phosphorus. J. Amer. Soc. Hort. Sci., v.117, p.721-724, 1992.

NOVAIS, R. F; SMYTH, T. J; NUNES, F. N. Fósforo. In: NOVAIS, R. F.et al. (Eds.). Fertilidade do solo. Viçosa: Sociedade Brasileira de Ciência do Solo, 2007. p.471-550.

OTANI, T; A. E. N. Phosphorus uptake mechanisms of crop grown in soil with low P status. I. Screening of crops for efficient P uptake. Soil Sci. Plant Nutr., v.42, p.155-163, 1996.

RAIJ, B. Van et al. Interpretação de resultados de análise de solo. In: RAIJ, B. van; CANTARELlA, H.; QUAGGIO, J. A; FURLANI, A.M.C.(Eds.). Recomendação de adubação e calagem para o Estado de São Paulo, Campinas: Fundação IAC, 1996. p.8-13.

RAIJ, B. Van et al. Análise química do solo para fins de fertilidade, Campinas: Fundação Cargill, 1987. $170 \mathrm{p}$. 
SANCHEZ, C. A; BURDINE, H. W. Relationship between lettuce yield and soil-test P and K levels. In: SOIL CROP SCIENCE SOCIETY FLORIDA. Proceeding... v.47, p.52-56, 1988.

SANCHEZ, C. A. et al. Yield, quality, and leaf nutrient composition of crisphead lettuce as affected by N, P, and K on Histosols. In: FLORIDA STATE HORTICULTURAL SCIENCE, Proceeding... v.101, p.346-350, 1988.

SANCHEZ, C. A; EL-HOUT, N. M. Response of diverse lettuce types to fertilizer phosphorus. HortScience, v.30, p.528-531, 1995.

SANCHEZ, C. A; SWANSON, S; PORTER, P. S. Banding phosphorus to improve fertilizer use efficiency of lettuce. J. Amer. Soc. Hortic. Sci., v.115, p.581-584, 1990.

SENO, S; KOGA, P. S. Efeitos de húmus de minhoca e fósforo na produção da alface (Lactuca sativa L.). Cultura Agronômica, v.2, p.129-139, 1993.

TRANI, P. E; PASSOS, F. A; AZEVEDO FILHO, J. A. Alface, almeirão, chicória, escarola, rúcula e agrião d'água. In: RAIJ, B. vanet al.(Eds.). Recomendação de adubação e calagem para o Estado de São Paulo, Campinas: Fundação IAC, 1996. p.168-169.

TRANI, P. E; RAIJ, B. Van. Hortaliças. In: RAIJ, B. van et al.(Eds.). Recomendação de adubação e calagem para o Estado de São Paulo, Campinas: Fundação IAC, 1996. p.157164

$\mathrm{XU}, \mathrm{G}$. et al. Integrated effect of irrigation frequency and phosphorus level on lettuce: $\mathrm{P}$ uptake, root growth and yield. Plant and Soil, v.263, p.297-309, 2004. 